\title{
ISOMETRICALLY INVARIANT EXTENSIONS OF LEBESGUE MEASURE
}

\author{
KRZYSZTOF CIESIELSKI
}

(Communicated by R. Daniel Mauldin)

\begin{abstract}
The purpose of this note is to give a very short prove of the theorem thta every isometrically invariant measure extending Lebesgue measure on $\mathbf{R}^{n}$ has a proper isometrically invariant extension, i.e., that there is no maximal isometrically invariant extension of Lebesgue measure on $\mathbf{R}^{n}$.
\end{abstract}

All the measures that we will consider in this note will be countable additive isometrically invariant extensions of Lebesgue measure on $n$-dimensional Euclidean space $\mathbf{R}^{n}$. By isometries we will understand bijections of $\mathbf{R}^{n}$ that preserve standard Euclidean distance. All the algebraic and measure theoretical terminology that will be used is standard and follows from [ $\mathrm{La}, \mathrm{Ru}]$ respectively.

The first construction of a proper isometrically invariant extension of Lebesgue measure goes back to Szpilrajn's paper [Sz] of 1935. In the same paper, Szpilrajn stated Sierpinski's question: "Does there exist a maximal isometrically invariant extension of Lebesgue measure on $\mathbf{R}^{n}$ ?" A negative answer to this question, i.e., the theorem "every isometrically invariant measure that extends Lebesgue measure on $\mathbf{R}^{n}$ has a proper isometrically invariant extension," was proved by several mathematicians under different additional assumptions and restrictions (see [ $\mathrm{Pk}, \mathrm{Hu}, \mathrm{Ha}]$ ). Without any assumption the theorem was proved in 1983 by Ciesielski and Pelc (see [CP]). For more historical details of this issue see also [Ci1]. The purpose of this note is to give a very short proof of the theorem that is different from that of [CP] and follows from the general technique introduced by the author in [Ci2].

Theorem. Let $\mu: \mathscr{M} \rightarrow[0, \infty]$ be an isometrically invariant extension of Lebesgue measure $\mathbf{R}^{n}$. Then there exists a proper isometrically invariant extension of $\mu$.

The proof will be based on the following easy and well-known lemmas.

Lemma 1 (Szpilrajn). Let $\mu: \mathscr{M} \rightarrow[0, \infty]$ be an isometrically invariant measure on $\mathbf{R}^{n}$. If a family $\mathscr{A}$ of subsets of $\mathbf{R}^{n}$ is closed under countable union,

Received by the editors October 30,1989; the content of this paper has been presented to Sixth Annual Auburn Miniconference on Real Analysis on April 7, 1989.

1980 Mathematics Subject Classification (1985 Revision). Primary 28C10.

This research was sponsored by the NSF. 
closed under isometries action (i.e., $g[A] \in \mathscr{A}$ for every $A \in \mathscr{A}$ and every isometry $g$ ) and such that every $A \in \mathscr{A}$ has $\mu$ inner measure zero, then $\mu$ has an isometrically invariant extension $\nu: \mathscr{N} \rightarrow[0, \infty]$ such that $\mathscr{A} \subset \mathscr{N}$ and $\nu(A)=0$ for every $A \in \mathscr{A}$.

Proof. If $\mathscr{I}$ is an ideal of subsets of $\mathbf{R}^{n}$ generated by the family $\mathscr{A}$, and $\mathscr{N}$ stands for a $\sigma$-algebra generated by $\mathscr{M} \cup \mathscr{F}$ then all elements of $\mathscr{N}$ are of the form $\left(M \cup I_{1}\right) \backslash I_{2}$ where $M \in \mathscr{M}$ and $I_{1}, I_{2} \in \mathscr{I}$. It is easy to see that $\nu: \mathcal{N} \rightarrow[0, \infty]$ such that $\nu\left(\left(M \cup I_{1}\right) \backslash I_{2}\right)=\mu(M)$ is a well-defined isometrically invariant measure on $\mathbf{R}^{n}$ extending $\mu$.

In the proof of the next lemma we use a method which goes back to Harazisvili's paper [Ha] (see also [Ci2]).

Lemma 2. Let $\mathbf{R}^{n}=\bigcup\left\{N_{k}: k=0,1,2, \ldots\right\}$. If each $N_{k}$ satisfies the condition for every countable set $\left\{g_{r}: r=0,1,2, \ldots\right\}$ of isometries there is an uncountable set $H$ of isometries such that for every distinct $h_{1}, h_{2} \in H$

$$
\begin{aligned}
& h_{1}\left(\bigcup\left\{g_{r}\left[N_{k}\right]: r=0,1,2, \ldots\right\}\right) \\
& \quad \cap h_{2}\left(\bigcup\left\{g_{r}\left[N_{k}\right]: r=0,1,2, \ldots\right\}\right)=\varnothing
\end{aligned}
$$

then every isometrically invariant extension $\mu: \mathscr{M} \rightarrow[0, \infty]$ of Lebesgue measure on $\mathbf{R}^{n}$ has a proper isometrically invariant extension.

Proof. Let $\mu: \mathscr{M} \rightarrow[0, \infty]$ be an isometrically invariant extension of Lebesgue measure on $\mathbf{R}^{n}$. Define

$$
\mathscr{A}_{k}=\left\{\bigcup\left\{g_{r}\left[N_{k}\right]: r=0,1,2, \ldots\right\}: \text { where all } g_{r} \text { 's areisometries of } \mathbf{R}^{n}\right\} \text {. }
$$

If $M \in \mathscr{M}$ is a subset of $A \in \mathscr{A}_{k}$ then $h_{1}[M] \cap h_{2}[M]=\varnothing$ for every distinct $h_{1}, h_{2}$ from $H$. But $\mu(h[M])=\mu(M)$ for every $h$ from $H$. Moreover, measure $\mu$ is $\sigma$-finite as an extension of Lebesgue measure. This implies that $\mu(M)=0$ and so $A$ has $\mu$ inner measure zero.

Thus we proved that every $\mathscr{A}_{k}$ satisfies the assumptions of Lemma 1 . Hence for each $k=0,1,2, \ldots$ there is an isometrically invariant extension $\nu_{k}$ of $\mu$ such that $\nu_{k}\left(N_{k}\right)=0$. But all $N_{k}$ 's cannot have $\mu$ measure zero. So some $\nu_{k}$ must be a proper extension of $\mu$.

The following lemma is an elementary geometrical fact and will be left without the proof.

Lemma 3. Every isometry of $\mathbf{R}^{n}$ can be represented as a superposition to $L$ where $t$ is a translation by a vector $\left(t_{1}, t_{2}, \ldots, t_{n}\right)$ and $L$ is a linear transformation of $\mathbf{R}^{n}$ represented by some $n \times n$ matrix $\left(a_{i j}\right)$.

Proof of the theorem. By Lemma 2 it is enough to construct $N_{k}$ 's such that $\mathbf{R}^{n}=\bigcup\left\{N_{k}: k=0,1,2, \ldots\right\}$ and each $N_{k}$ satisfies condition $(*)$. 
Let $\mathscr{B}$ be a transcendence base of $\mathbf{R}$ over $\mathbf{Q}$ and let us represent $\mathscr{B}$ as $\mathscr{B}=\bigcup\left\{\mathscr{B}_{k}: k=0,1,2, \ldots\right\}$ where $\mathscr{B}_{0} \subset \mathscr{B}_{1} \subset \mathscr{B}_{2} \subset \cdots$ and $\mathscr{B}_{k+1} \backslash \mathscr{B}_{k}$ is uncountable. Define

$$
N_{k}=\left[\mathrm{cl}_{\mathbf{R}}\left(\mathbf{Q}\left(\mathscr{B}_{k}\right)\right)\right]^{n},
$$

where $\mathbf{Q}\left(\mathscr{B}_{k}\right)$ is a field generated by $\mathbf{Q}$ and $\mathscr{B}_{k}$ and $\operatorname{cl}_{\mathbf{R}}\left(\mathbf{Q}\left(\mathscr{B}_{k}\right)\right)$ is an algebraic closure of $\mathbf{Q}\left(\mathscr{B}_{k}\right)$ in $\mathbf{R}$. We have to prove that $N_{k}$ 's satisfy $(*)$.

So let us choose $k$ and a countable set $\left\{g_{r}: r=0,1,2, \ldots\right\}$ of isometries. There exists a countable set $\mathscr{A} \subset \mathscr{B}$ such that all $g_{r}$ 's are defined over $\left.\mathrm{cl}_{\mathbf{R}}(\mathbf{Q} \mathscr{A})\right)$, i.e., that for each $g_{r}$ the coefficients $t_{i}$ 's and $a_{i j}$ 's from Lemma 3 are in $\operatorname{cl}_{\mathbf{R}}(\mathbf{Q}(\mathscr{A}))$. Let $L=\operatorname{cl}_{\mathbf{R}}\left(\mathbf{Q}\left(\mathscr{A} \cup \mathscr{B}_{k}\right)\right)$. Then

$$
\bigcup\left\{g_{r}\left[N_{k}\right]: r=0,1,2, \ldots\right\} \subset L^{n} .
$$

Define

$$
H=\left\{t_{\alpha}: \alpha \in \mathscr{B}_{k+1} \backslash\left(\mathscr{A} \cup \mathscr{B}_{k}\right)\right\},
$$

where $t_{\alpha}$ is a translation by a vector $(\alpha, 0,0, \ldots, 0)$. Then $H$ is uncountable and for distinct $\alpha, \beta \in H$,

$$
\begin{gathered}
t_{\alpha}\left(\bigcup\left\{g_{r}\left[N_{k}\right]: r=0,1,2 \ldots\right\}\right) \cap t_{\beta}\left(\bigcup\left\{g_{r}\left[N_{k}\right]: r=0,1,2, \ldots\right\}\right) \\
\subset t_{\alpha}\left(L^{n}\right) \cap t_{\beta}\left(L^{n}\right)=\varnothing
\end{gathered}
$$

as $\alpha-\beta \notin L$. This finishes the proof of the theorem.

\section{REFERENCES}

[Ci1] K.Ciesielski, How good is Lebesgue measure?, Math. Intelligencer 11 (1989), 54-58.

[Ci2] _ Algebraically invariant extensions of $\sigma$-finite measures on Euclidean space, Trans. Amer. Math. Soc. 315 (1989).

[CP] K. Ciesielski and A. Pelc, Extensions of invariant measures on Eucledean spaces, Fund. Math. 125 (1985), 1-10.

[Ha] A. B. Harazisvili, On Sierpinski's problem concerning strict extendibility of an invariant measure, Soviet Math. Dokl. 81 (1977), 71-74.

[Hu] A. Hulanicki, Invariant extensions of the Lebesgue measure, Fund. Math. 51 (1962), 111-115.

[La] S. Lang, Algebra, Addison-Wesley, 1984.

[Pk] S. S. Pkhakadze, $K$ teorii lebegovskoi miery, Trudy Tbiliss. Mat. Inst. Razmadze Akad. Nauk Gruzin. SSR, vol. 25, 1958. (Russian)

[Ru] W. Rudin, Real and complex analysis, McGraw-Hill, 1987.

[Sz] E. Szpilrajn, Sur l'extension de la mesure lebesguienne, Fund. Math. 25 (1935), 551-558. (French)

Department of Mathematics, West Virginia University, Morgantown, West Virginia 